\title{
Interaction between a galactic disk and a live dark halo with an anisotropic velocity distribution (Research Note)
}

\author{
B. Fuchs ${ }^{1}$ and E. Athanassoula ${ }^{2}$
}

\author{
1 Astronomisches Rechen-Institut, Mönchhofstrasse 12-14, 69120 Heidelberg, Germany \\ e-mail: fuchs@ari.uni-heidelberg.de \\ 2 Observatoire de Marseille, 2 Place Le Verrier, 13248 Marseille Cedex 04, France
}

Received 17 February 2005 / Accepted 21 September 2005

\begin{abstract}
We have extended previous analytical studies of the interaction of dark halos with galactic disks by introducing for the halo particles anisotropic distribution functions in phase space. For this purpose we have employed the shearing sheet model of a patch of a galactic disk embedded in a homogeneous halo. We find that velocity anisotropy increases considerably the maximum growth factor of perturbations in the disk.
\end{abstract}

Key words. galaxies: kinematics and dynamics - galaxies: spiral

\section{Introduction}

The dynamical evolution of non-axisymmetric structures such as spirals or bars is driven by the redistribution of angular momentum within the disk galaxy that harbours them. This was argued and demonstrated by Lynden-Bell \& Kalnajs (1972), who showed that stars at inner Lindblad resonance and at higher order resonances within corotation emit angular momentum, which is absorbed by stars at corotation, at outer Lindblad resonance and at higher order resonances outside corotation. Nonresonant stars can also contribute to this exchange, provided the spiral or bar perturbation has a non-negligible growth rate. Since the non-axisymmetric structures are inside the corotation circle negative angular momentum "perturbations" (Kalnajs 1971; Lynden-Bell \& Kalnajs 1972), this exchange induces their growth. In this early picture the role of the spheroid is not included. However, recent, state-of-the-art $N$-body simulations have clearly shown that it can be predominant in galaxies with strong bars. Indeed, if the galactic disk is embedded in a spheroid or dark halo, a similar angular momentum exchange between the bar and the surrounding bulge or dark halo is expected. Athanassoula $(2002,2003)$ studied in particular the interaction of a bar in the disk and a live spheroidal component (bulge and/or halo). Provided the latter has a distribution function that is a function of the energy only, it is possible to show analytically that it can at all its resonances only absorb angular momentum. Thus in the presence of spheroid or a halo a bar can grow stronger than in its absence, since in the former case more angular momentum is taken from the bar region. Tremaine \& Weinberg (1984) and Weinberg (1985) calculated that the angular momentum exchange also causes a considerable slow-down of the bar, which is similarly enhanced in the presence of a live spheroid with an isotropic distribution function. This was, at least qualitatively, confirmed by a number of $N$-body simulations (e.g. Little \& Carlberg 1991; Hernquist \& Weinberg 1992; Athanassoula 1996; Debattista \& Sellwood 2000; Athanassoula 2003; O’Neill \& Dubinski 2003; Valenzuela \& Klypin 2003). Athanassoula (2002, 2003) used $N$-body simulations to show that a considerable amount of halo material is at resonance with the bar. She further traced the angular momentum exchange in the simulations and found very good qualitative agreement with analytical predictions. Angular momentum is emitted by particles within corotation: mainly by particles at inner Lindblad resonance, but also by particles at higher order resonances and also by non-resonant particles. It is then absorbed by disk particles at corotation and outer Lindblad resonance, halo resonant particles at all resonances and non-resonant halo particles. Indeed, bars in simulations with a responsive halo, that can take part in the angular momentum exchange, grow considerably stronger than bars in simulations with a halo which has the same mass distribution, but is rigid (Athanassoula 2002). This justifies, in retrospect, the doubts voiced much earlier by Toomre (1977) as to the entirely passive role of the halo in bar formation and development.

The dynamics of bars and spiral density waves in galactic disks are closely related to each other (Toomre 1981; Bertin et al. 1989a,b). Accordingly enhanced growth due to 
the presence of a responsive halo is also known for spiral density waves. Mark (1976b) has studied the angular momentum transfer from mode like spiral density waves to a bulge-halo system surrounding the galactic disk. The density waves were modelled as rigidly rotating exponentially growing spiral patterns whose dynamics was described in the WKBJ approximation (Mark 1974; 1976a). The models presented in Mark (1976b, hereafter referred to as M76) show considerably enhanced growth rates of the density waves due to angular momentum loss to the bulge - halo system. Recently Fuchs (2004, hereafter referred to as F04) followed a different approach to the spiral amplification question in the presence of a live dark halo. Using the shearing sheet model he showed how a live dark halo responds to swing amplified spiral density waves and found also a very enhanced growth of such density waves. These density waves interact with the halo via halo particles either on orbits in resonance with the waves or on non-resonant orbits.

The shearing sheet model (Goldreich \& Lynden-Bell 1965; Julian \& Toomre 1966) has been developed as a tool to study the dynamics of a patch of a galactic disk. This is assumed to be infinitesimally thin and its radial size is assumed to be much smaller than that of the disk. Polar coordinates can be therefore rectified to pseudo-Cartesian coordinates and the velocity field of the differential rotation of the disk can be approximated by a linear shear flow. These simplifications allow an analytical treatment of the problem, which helps to clarify the underlying physical processes operating in the disk.

Up to now all studies of the effect of a live dark halo on the dynamics of non-axisymmetric structures in a galactic disk have assumed an isotropic velocity distribution of the halo particles. On the other hand, it is well known from cosmological simulations of dark halo formation that the velocity distribution of the halo particles must be anisotropic. If the anisotropy is measured by Binney's parameter $\beta=1-\left(\sigma_{\theta}^{2}+\sigma_{\phi}^{2}\right) / 2 \sigma_{r}^{2}$ (cf. Binney \& Tremaine 1987) this ranges from $\beta \approx 0$ near the centers of the halos to $\beta \approx 0.5$ at the virial radii (Cólin et al. 2000; Fukushige \& Makino 2001). Hansen \& Moore (2005) have suggested that the radial variation of $\beta$ is roughly linear to the logarithmic density gradient of the halo, $\beta \propto-\mathrm{d} \ln \rho / \mathrm{d} \ln r$. Very recently Abadi et al. (2005) have reported a simulation of a mildly anisotropic dark halo with an anisotropy parameter of $\beta \approx 0.3$. In the same experiment they have also simulated the formation of the baryonic galaxy inside the dark halo. Interestingly, the velocity anisotropy of the spheroidal component of the model galaxy is significantly larger, $\beta \approx 0.4$ to 0.8 , which is consistent with the anisotropy parameter of $\beta \approx 0.7$ of Galactic halo stars in the solar neighbourhood (Arifyanto et al. 2005). Following a different concept, which describes dark halos as partially relaxed dynamical systems, Trenti \& Bertin (2005) find also radially anisotropic velocity distributions with an anisotropy parameter in the region around corotation of $\beta \approx 0.6$. Helmi et al. (2002) discuss the implications of such an anisotropy for experimental searches of dark matter particles.

Thus the studies of the interaction of dark halos with nonaxisymmetric structures in galactic disks have to be extended to halo models with anisotropic velocity distributions. As a first step we consider in this short note the amplification of spiral density waves in disks embedded in anisotropic dark halos.

\section{Introducing anisotropic distribution functions for the halo particles}

\subsection{Response of a live dark halo to a density wave in the shearing sheet}

We study now the response of a live dark halo with an anisotropic velocity distribution to a swing amplified density wave in the disk. For this we use the method and notation of F04, assuming that the shearing sheet is immersed in a homogeneous live dark halo. Since we modify only the unperturbed distribution function of the halo particles in phase space, we can immediately follow the formalism of F04 to derive the Fourier transformed Boltzmann equation which describes the evolution of the perturbation of the distribution function $f_{\mathrm{h} k}$ (Eq. (20) of F04). We adopt now the anisotropic background distribution function

$f_{\mathrm{h} 0}=\frac{\rho_{\mathrm{b}}}{\sqrt{2 \pi^{3} \sigma_{\|}^{2} \sigma_{w}}} \exp \left(-\frac{u^{2}+v^{2}}{2 \sigma_{\|}^{2}}-\frac{w^{2}}{2 \sigma_{w}^{2}}\right)$.

$u$ and $v$ denote the planar velocity components of the halo particles with $v$ pointing in the direction of the differential shear in the sheet. $w$ is the velocity component perpendicular to the plane of the sheet. As in F04 we choose orthogonal Cartesian coordinates $\xi, \eta$, and $\zeta$ with $\xi$ parallel to the wave vector $\boldsymbol{k}$ of $f_{\mathrm{h} k}$. According to the symmetry of the distribution function (1), one of the coordinates perpendicular to $\boldsymbol{k}$ can be aligned without loss of generality with the $v$ velocity component. Thus the distribution function (1) can be written as

$$
\begin{aligned}
f_{\mathrm{h} 0}= & \frac{\rho_{\mathrm{b}}}{\sqrt{2 \pi}^{3} \sigma_{\|}^{2} \sigma_{w}} \exp \left(-\frac{v^{2}}{2 \sigma_{\|}^{2}}-\frac{\left(v_{\|} \cos \alpha-v_{\perp} \sin \alpha\right)^{2}}{2 \sigma_{\|}^{2}}\right) \\
& \times \exp \left(-\frac{\left(v_{\|} \sin \alpha+v_{\perp} \cos \alpha\right)^{2}}{2 \sigma_{w}^{2}}\right),
\end{aligned}
$$

where $\cos \alpha=k_{\|} / \sqrt{k_{z}^{2}+k_{\|}^{2}}$, and $k_{\|}=\left|\boldsymbol{k}_{\|}\right|=\sqrt{k_{x}^{2}+k_{y}^{2}}$ is the component of the wave vector $\boldsymbol{k}$ parallel to the mid-plane of the sheet. $v_{\perp}$ denotes the second velocity component perpendicular to $\boldsymbol{k}$. The Boltzmann equation can be then immediately integrated with respect to $v$ and, after some algebra, the integration with respect to $v_{\perp}$ leads to a Boltzmann equation which has the same form as Eq. (20) of F04, but where the one-dimensional velocity dispersion $\sigma_{\mathrm{h}}$ of the isotropic distribution function is replaced by the velocity dispersion of the velocity component in the $\xi$-direction parallel to $\boldsymbol{k}$

$\sigma_{\mathrm{eff}}=\sqrt{\sigma_{\|}^{2} \cos \alpha^{2}+\sigma_{w}^{2} \sin \alpha^{2}}$

We can proceed then exactly as in F04 and obtain the same final formal result $\Phi_{\mathrm{h} k_{\|}}(z=0)=\Upsilon\left(k_{x}, k_{y}, \omega\right) \Phi_{\mathrm{d} k_{\|}}$, where the function $\Upsilon$ is modified by replacing $\sigma_{\mathrm{h}}$ by $\sigma_{\text {eff }}$ in Eqs. (15) and (17) of F04. 
As alternatives to the distribution function of the halo particles (1) we have considered also the anisotropic distribution functions

$f_{\mathrm{h} 0}=\frac{\rho_{\mathrm{b}}}{\sqrt{2 \pi^{3}} \sigma_{u} \sigma_{w}^{2}} \exp \left(-\frac{u^{2}}{2 \sigma_{u}^{2}}-\frac{v^{2}+w^{2}}{2 \sigma_{w}^{2}}\right)$

and

$f_{\mathrm{h} 0}=\frac{\rho_{\mathrm{b}}}{\sqrt{2 \pi^{3}} \sigma_{v} \sigma_{w}^{2}} \exp \left(-\frac{v^{2}}{2 \sigma_{v}^{2}}-\frac{u^{2}+w^{2}}{2 \sigma_{w}^{2}}\right)$.

If these are integrated with respect to the two velocity components perpendicular to $\boldsymbol{k}$, we find the effective velocity dispersions

$\sigma_{\mathrm{eff}}=\sqrt{\sigma_{u}^{2} \cos \beta^{2}+\sigma_{w}^{2} \sin \beta^{2}}$

and

$\sigma_{\text {eff }}=\sqrt{\sigma_{v}^{2} \cos \gamma^{2}+\sigma_{w}^{2} \sin \gamma^{2}}$,

respectively. The angles $\beta$ and $\gamma$ are given by $\cos \beta=$ $k_{x} / \sqrt{k_{z}^{2}+k_{\|}^{2}}$ and $\cos \gamma=k_{y} / \sqrt{k_{z}^{2}+k_{\|}^{2}}$. An effective velocity dispersion $\sigma_{\text {eff }}$ can also be calculated analytically for a triaxial distribution function. However, the result is so cumbersome that we do not consider this case here.

\subsection{Dynamics of the shearing sheet embedded in an anisotropic live dark halo}

The halo response to the perturbation in the disk has to be inserted into the Boltzmann equation describing the evolution of the distribution function of the disk particles in phase space. We follow again the formalism of F04 and find the fundamental Volterra type integral equation for the Fourier transforms of the perturbations of the gravitational potential of the shearing sheet in the same form as Eq. (33) of F04, but with a modified Fourier transform $\mathcal{F}\left(\Upsilon \mathrm{e}^{\mathrm{i} \omega \frac{k_{x}-k_{x}^{\prime}}{2 A k_{y}}}\right)_{t-t^{\prime}}$. The latter can be calculated analytically as in F04 using first formulae 6.317 and 3.952 of Gradshteyn \& Ryzhik (2000) and then formulae 3.223 and 3.466 for the integrals with respect to $k_{z}$, leading to

$$
\begin{aligned}
& \mathcal{F}\left(\Upsilon\left(k_{x}, k_{y}^{\prime}, \omega\right) \mathrm{e}^{\mathrm{i} \omega \frac{k_{x}-k_{x}^{\prime}}{2 A k_{y}}}\right)_{t-t^{\prime}}= \\
& \quad \frac{8 \pi^{2} G \rho_{\mathrm{b}}}{\sigma_{\|}\left(\sigma_{\|}+\sigma_{w}\right)} \frac{1}{k_{\mathrm{x}}^{2}+k_{y}^{\prime 2}} \delta\left(t-t^{\prime}+\frac{k_{x}-k_{x}^{\prime}}{2 A k_{y}^{\prime}}\right) \\
& \quad+4 \pi^{2} G \rho_{\mathrm{b}} \exp \left[\mathrm{i} k_{y}^{\prime} r_{0} \Omega_{0}\left(t-t^{\prime}+\frac{k_{x}-k_{x}^{\prime}}{2 A k_{y}^{\prime}}\right)\right] \\
& \quad \times\left\{\left(t-t^{\prime}+\frac{k_{x}-k_{x}^{\prime}}{2 A k_{y}^{\prime}}\right)+\left|t-t^{\prime}+\frac{k_{x}-k_{x}^{\prime}}{2 A k_{y}^{\prime}}\right|\right\} \\
& \quad \times \exp \left[-\frac{1}{2}\left(\sigma_{\|}^{2}-\sigma_{w}^{2}\right)\left(k_{x}^{2}+k_{x}^{\prime 2}\right)\left(t-t^{\prime}+\frac{k_{x}-k_{x}^{\prime}}{2 A k_{y}^{\prime}}\right)^{2}\right] \\
& \left.\quad \times \operatorname{erfc}\left(\frac{\sigma_{w}}{\sqrt{2}} \sqrt{k_{x}^{2}+k_{y}^{\prime 2}} \mid t-t^{\prime}+\frac{k_{x}-k_{x}^{\prime}}{2 A k_{y}^{\prime}}\right)\right) .
\end{aligned}
$$

The modified Eq. (35) of F04 can be integrated numerically still with very modest numerical effort. The characteristic response of the shearing sheet embedded in a live dark halo to an initial impulse is not changed qualitatively by the anisotropy of the velocity distribution of the halo particles. The shearing sheet develops swing amplified density waves with their amplitudes enhanced by the presence of a live dark halo as compared to a static halo (cf. Fig. 2 of F04). In the case of the distribution function (4) we obtain the Fourier transform

$$
\begin{aligned}
& \mathcal{F}\left(\Upsilon\left(k_{x}, k_{y}^{\prime}, \omega\right) \mathrm{e}^{\mathrm{i} \omega \frac{k_{x}-k_{x}^{\prime}}{2 A k_{y}}}\right)_{t-t^{\prime}}= \\
& \frac{8 \pi^{2} G \rho_{\mathrm{b}}}{\sqrt{\sigma_{u}^{2} k_{x}^{2}+\sigma_{w}^{2} k_{y}^{\prime 2}}\left(\sigma_{w} \sqrt{k_{x}^{2}+k_{y}^{\prime 2}}+\sqrt{\sigma_{u}^{2} k_{x}^{2}+\sigma_{w}^{2} k_{y}^{\prime 2}}\right)} \\
& \times \delta\left(t-t^{\prime}+\frac{k_{x}-k_{x}^{\prime}}{2 A k_{y}^{\prime}}\right) \\
& +4 \pi^{2} G \rho_{\mathrm{b}} \exp \left[i k_{y}^{\prime} r_{0} \Omega_{0}\left(t-t^{\prime}+\frac{k_{x}-k_{x}^{\prime}}{2 A k_{y}^{\prime}}\right)\right] \\
& \times\left\{\left(t-t^{\prime}+\frac{k_{x}-k_{x}^{\prime}}{2 A k_{y}^{\prime}}\right)+\left|t-t^{\prime}+\frac{k_{x}-k_{x}^{\prime}}{2 A k_{y}^{\prime}}\right|\right\} \\
& \quad \times \exp \left[-\frac{1}{2}\left(\sigma_{u}^{2}-\sigma_{w}^{2}\right) k_{x}^{2}\left(t-t^{\prime}+\frac{k_{x}-k_{x}^{\prime}}{2 A k_{y}^{\prime}}\right)^{2}\right] \\
& \left.\quad \times \operatorname{erfc}\left(\frac{\sigma_{w}}{\sqrt{2}} \sqrt{k_{x}^{2}+k_{y}^{\prime 2}} \mid t-t^{\prime}+\frac{k_{x}-k_{x}^{\prime}}{2 A k_{y}^{\prime}}\right)\right),
\end{aligned}
$$

and similarly for the distribution function (5)

$$
\begin{aligned}
& \mathcal{F}\left(\Upsilon\left(k_{x}, k_{y}^{\prime}, \omega\right) \mathrm{e}^{\mathrm{i} \omega \frac{k_{x}-k_{x}^{\prime}}{2 A k_{y}^{\prime}}}\right)_{t-t^{\prime}}= \\
& \frac{8 \pi^{2} G \rho_{\mathrm{b}}}{\sqrt{\sigma_{w}^{2} k_{x}^{2}+\sigma_{v}^{2} k_{y}^{\prime 2}}\left(\sigma_{w} \sqrt{k_{x}^{2}+k_{y}^{\prime 2}}+\sqrt{\sigma_{w}^{2} k_{x}^{2}+\sigma_{v}^{2} k_{y}^{\prime 2}}\right)} \\
& \times \delta\left(t-t^{\prime}+\frac{k_{x}-k_{x}^{\prime}}{2 A k_{y}^{\prime}}\right) \\
& +4 \pi^{2} G \rho_{\mathrm{b}} \exp \left[\mathrm{i} k_{y}^{\prime} r_{0} \Omega_{0}\left(t-t^{\prime}+\frac{k_{x}-k_{x}^{\prime}}{2 A k_{y}^{\prime}}\right)\right] \\
& \times\left\{\left(t-t^{\prime}+\frac{k_{x}-k_{x}^{\prime}}{2 A k_{y}^{\prime}}\right)+\left|t-t^{\prime}+\frac{k_{x}-k_{x}^{\prime}}{2 A k_{y}^{\prime}}\right|\right\} \\
& \quad \times \exp \left[-\frac{1}{2}\left(\sigma_{v}^{2}-\sigma_{w}^{2}\right) k_{y}^{\prime 2}\left(t-t^{\prime}+\frac{k_{x}-k_{x}^{\prime}}{2 A k_{y}^{\prime}}\right)^{2}\right] \\
& \left.\quad \times \operatorname{erfc}\left(\frac{\sigma_{w}}{\sqrt{2}} \sqrt{k_{x}^{2}+k_{y}^{\prime 2}} \mid t-t^{\prime}+\frac{k_{x}-k_{x}^{\prime}}{2 A k_{y}^{\prime}}\right)\right) .
\end{aligned}
$$

The characteristic response of the shearing sheet embedded in dark halos with these anisotropic distribution functions of the halo particles to an initial impulse is still the same as in the previous case, only the maximum growth factors of the density waves change. All three Fourier transforms (8-10) are reduced to the form given in F04, if the distribution functions become isotropic. 


\subsection{Numerical applications}

\subsubsection{Comparison with previous work}

No results with anisotropic halos, either analytic or numerical, have been published so far. Indeed, as mentioned already in the introduction, the lack of such information has been one of the main motivations for our work. A couple of previous works, however, have studied the influence of a live dark halo with an isotropic distribution function on density waves and we will present here some possible, albeit rough, comparisons.

The interaction of a WKBJ type density wave with a separate non-rotating component was studied for the first time by Marochnik \& Suchkov (1969). The astronomical community was at that time not aware of the existence of dark halos, but the considerations of Marochnik \& Suchkov (1969) carry over to the disk - dark halo interaction. Unfortunately, only very few concrete details were given. Much more detailed is the investigation by Mark (M76). In Fig. 3 of M76 it is shown how much stronger the amplification of the density waves is in the presence of a live halo than in an isolated disk. This depends on the assumed corotation radii of the spiral patterns. Since swing amplified density waves are basically corotating with the center of the shearing sheet, we adopt for each corotation radius of the spiral modes the parameters given in Table 1 of M76 for that galactocentric radius and calculate for these parameters the corresponding maximum growth factors of swing amplified density waves in the shearing sheet. The regions in the galaxy models, where the rotation curves are essentially flat, are most suitable for a comparison. Using the formalism discussed in the previous sections, we find for the parameters of model $A_{1}$ at $R=6 \mathrm{kpc}$ an enhancement of the maximum growth factor of the amplitudes of swing amplified density waves due to the halo by a factor of 2, while Fig. 3 of M76 indicates that the growth rates of the density waves are enhanced by a factor of 2.3. Similarly, we find for the parameters of model $\mathrm{B}_{1}$ in the range $R=14$ to $18 \mathrm{kpc}$ maximum growth factors enhanced by factors of 3.2 to 1.4. Figure 3 of M76 indicates for that range an enhancement of the growth rates by a factor of 1.7. Thus the numerical values are in rough agreement.

\subsubsection{Numerical applications to anisotropic halos}

We have determined maximum growth factors of the amplitudes of the density waves for various values of the parameters characterizing the disk - halo configuration. Representative results for the three cases described above are presented in Fig. 1. In all three cases we have taken $\sigma_{\text {tot }}^{2}=\sigma_{u}^{2}+\sigma_{v}^{2}+\sigma_{w}^{2}=1$, $A / \Omega_{0}=0.5$ and $k_{y}^{\prime}=0.5 k_{\text {crit. }}$ In F04 it was shown how Eqs. (8) to (10) can be cast into dimensionless form. The velocity dispersion of the halo particles is then measured in terms of the velocity dispersion of the disk stars. We have assumed for the examples in Fig. 1 a ratio of $\sigma_{\text {tot }}: \sigma_{\text {disk }}=5: 1$. The velocity dispersion of the disk stars is determined by the Toomre stability parameter for which we have adopted a value of $Q=1.41$. For the density of the dark halo and the circular velocity of the center of the shearing sheet around the galactic center we have adopted values typical for the solar neighbourhood in the

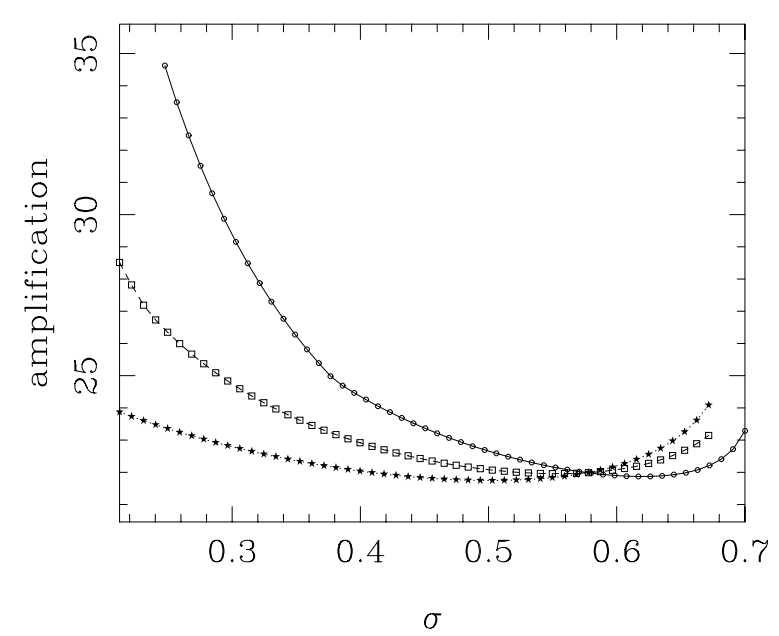

Fig. 1. Maximum growth factor of the amplitudes of density waves in the shearing sheet as function of the anisotropy of the velocity distribution of the halo particles. The solid line with open circles illustrates the case if the components of the velocity dispersion are varied as $\sigma_{u}=\sigma_{v}$. These are drawn as abscissa. The third component is chosen as $\sigma_{w}=\sqrt{1-2 \sigma_{u}^{2}}$, so that the total velocity dispersion is constant. The cases $\sigma_{v}=\sigma_{w}, \sigma_{u}=\sqrt{1-2 \sigma_{v}^{2}}$ are shown as the dashed line with open squares and $\sigma_{u}=\sigma_{w}, \sigma_{v}=\sqrt{1-2 \sigma_{u}^{2}}$ as the dotted line with filled asterisks, respectively. The isotropic case $\sigma=0.58$ reproduces the result of $\mathrm{F} 04$.

Milky Way, namely $\rho_{\mathrm{b}} G / \kappa^{2}=0.01$ and $r_{0} \Omega_{0}: \sigma_{\text {disk }}=220$ : 44. We find in all three cases the same characteristic behaviour, that the amplification of density waves in the shearing sheet is smallest, if the distribution function of the halo particles is almost, but not exactly, isotropic. Even in the isotropic case, though, the amplification is still considerably larger than in a shearing sheet embedded in a static halo. The dependence of the maximum growth factor on the velocity dispersion components is rather subtle. If we concentrate on the case $\sigma_{u}=\sigma_{v}$, it becomes clear from Eq. (8) that $\mathcal{F}$ rises, if $\sigma_{\|}$is decreasing with respect to $\sigma_{w}$, which leads to a stronger input to the swing amplification mechanism. This can be intuitively understood as the halo becomes more susceptible to the sheared trailing spiral arms in the disk, if the planar velocity dispersions become small. The dependence of $\mathcal{F}$ on the $\sigma_{w}$ velocity dispersion component is twofold. Technically, this is related to the integrations of the halo response to the disk perturbations with respect to the $k_{z}$ wave number (cf. Eqs. (26) and (28) of F04). The result is that $\mathcal{F}$ rises also at lower $\sigma_{w}$ velocity dispersions. This means that the dark halo becomes then less stiff in the vertical direction so that it can support the density waves in the disk more effectively, although the effect is less pronounced. The relative minimum of amplification of density waves is at slightly larger $\sigma_{u}=\sigma_{v}$ velocity dispersions than the isotropic case $\sigma_{u}=\sigma_{v}=\sigma_{w}=0.58 \sigma_{\text {tot }}$. The other diagrams in Fig. 1 can be explained by similar arguments. However, since in these cases only one planar velocity dispersion is varied, the maximum growth factor is less than in the previous case. We have also considered other numerical values for the parameters $Q$ and $A$ of the disk as well as for the halo parameters $\rho_{\mathrm{b}}$ and $\sigma_{\text {tot }}$. Even though the overall amplification of the density waves can 
change drastically, the effect of the anisotropy as compared to the isotopic case remains always roughly the same.

Extrapolating these results, we predict that spirals and bars growing in disk galaxies with non-isotropic halos should be on average stronger than those in isotropic halos. Unfortunately it is not possible to test this for real galaxies. It is, however, possible to do so for $\mathrm{N}$-body simulations and such a study is presently underway (Athanassoula, in prep.).

Acknowledgements. We thank the anonymous referee for his comments which helped to improve the paper.

\section{References}

Abadi, M. G., Navarro, J., \& Steinmetz, M. 2005, [arXiv: astr-ph/0506659]

Arifyanto, M. I., Fuchs, B., Jahreiß, H., \& Wielen, R. 2005, A\&A, 433, 911

Athanassoula, E. 1996, in Barred Galaxies, Astron. Soc. Pac. Conf. Ser., ed. R. Buta, D. A. Crocker, \& Elmegreen, B. G., 91, 309

Athanassoula, E. 2002, ApJ, 569, L83

Athanassoula, E. 2003, MNRAS, 341, 1179

Binney, J., \& Tremaine, S. 1987, Galactic Dynamics (Princeton: Princeton University Press)

Bertin, G., Lin, C. C., Lowe, S. A., \& Thurstans, R. P. 1989a, ApJ, 338,78

Bertin, G., Lin, C. C., Lowe, S. A., \& Thurstans, R. P. 1989b, ApJ, 338,104
Cólin, P., Klypin, A. A., \& Kravtsov, A. V. 2000, ApJ, 539, 561

Debattista, V. P., \& Sellwood, J. A. 2000, ApJ, 543, 704

Fuchs, B. 2004, A\&A, 419, 941

Fukushige, T., \& Makino, J. 2001, 557, 533

Goldreich, P., \& Lynden-Bell, D. 1965, MNRAS, 130, 125

Gradshteyn, I. S., \& Ryzhik, I. M. 2000, Table of Integrals, Series, and Products, 6th edn. (New York: Academic Press)

Hansen, S., \& Moore, B. 2005, [arXiv: astro-ph/04011473]

Helmi, A., White, S. D. M., \& Springel, V. 2002, PRD, 66, 063502

Hernquist, L., \& Weinberg, M. 1992, ApJ, 400, 80

Julian, W. H., \& Toomre, A. 1966, ApJ, 146, 810

Kalnajs, A. J. 1971, ApJ, 166, 275

Little, B., \& Carlberg, R. G. 1991, MNRAS, 251, 227

Lynden-Bell, D., \& Kalnajs, A. J. 1972, MNRAS, 157, 1

Mark, J. W. K. 1974, ApJ, 193, 539

Mark, J. W. K. 1976a, ApJ, 205, 363

Mark, J. W. K. 1976b, ApJ, 206, 418

Marochnik, L. S., \& Suchkov, A. A. 1969, AZ, 46, 319

O'Neill, J. K., \& Dubinski, J. 2003, MNRAS, 346, 251

Toomre, A. 1977, ARA\&A, 15, 437

Toomre, A. 1981, in The Structure and Evolution of Normal Galaxies, ed. S. M. Fall, \& D. Lynden-Bell (Cambridge: Cambridge Univ. Press), 111

Tremaine, S., \& Weinberg, M. D. 1984, MNRAS, 209, 729

Trenti, M., \& Bertin, G. 2005, A\&A, 429, 161

Valenzuela, O., \& Klypin, A. 2003, MNRAS, 345, 406

Weinberg, M. D. 1985, MNRAS, 213, 451 